\begin{tabular}{|c|c|c|c|}
\hline & $\begin{array}{l}\text { REUISTA DE EST } \\
\text { ALBACETEUS }\end{array}$ & $\begin{array}{c}\text { Número } \\
14\end{array}$ & $\begin{array}{l}\text { Páginas } \\
43-60\end{array}$ \\
\hline
\end{tabular}

\title{
EFECTO DE LOS TRABAJOS POST-INCENDIO SOBRE EL SUELO Y LA VEGETACION EN LA SIERRA DE LOS DONCELES (HELLIN, ALBACETE)
}

\section{THE EFFECT OF POST-FIRE WORK ON SOIL AND VEGETATION IN THE SIERRA DE LOS DONCELES (HELLÍN, ALBACETE)}

\author{
Por \\ Manuel Esteban LUCAS-BORJA ${ }^{1, *}$ \\ Elena GÓMEZ-SÁNCHEZ ${ }^{2}$ \\ Pedro Antonio PLAZA-ALVAREZ ${ }^{1}$ \\ Javier GONZÁLEZ-ROMERO ${ }^{1}$ \\ Javier SAGRA ${ }^{1}$ \\ Daniel MOYA ${ }^{1}$ \\ Jorge DE LAS HERAS ${ }^{1}$
}

Recibido: 19 de septiembre de 2019

Aprobado: 30 de octubre de 2020

\footnotetext{
${ }^{1}$ Escuela Técnica Superior de Ingenieros Agrónomos y de Montes. Universidad de Castilla La Mancha, Campus Universitario s/n. 02071. Albacete

${ }^{2}$ Servicio de Montes y Espacios Naturales Protegidos. Servicios Periféricos de la Consejería de Agricultura en Albacete. Junta de Comunidades de Castilla la Mancha. C/Mayor 4702071 . Albacete.

^ManuelEsteban.Lucas@uclm.es

Cómo citar este artículo:

Lucas-Borja, M.E., Gómez-Sánchez, E., Plaza-Álvarez, P.A., González-Romero, J., Sagra, J., Moya, D., De las Heras, J. (2020). Efecto de los trabajos post-incendio sobre el suelo y la vegetación en la Sierra de Los Donceles (Hellin, Albacete). Sabuco, 14: 43-60 http://doi.org/10.37927/sabuco.14_2
} 



\section{RESUMEN}

Las actuaciones en materia de restauración post-incendio engloban trabajos destinados a minimizar los impactos negativos del fuego, así como la rehabilitación ecológica de la zona quemada. El suelo y la vegetación se ven muy afectados y son muy vulnerables al paso del fuego, tanto por los efectos directos, como por los procesos erosivos que origina. El presente trabajo pretende evaluar el efecto que las técnicas de restauración post-incendio utilizadas a nivel de ladera (acordonado y fajinas) tienen sobre la funcionalidad del suelo y la recuperación de la vegetación 5 años después del incendio. Los trabajos de restauración se aplicaron entre 3 y 5 meses después del incendio y se comparan en este trabajo zonas de acordonado, fajinas, quemado sin actuación y no quemado (4 zonas con 3 réplicas cada una). Los resultados indican que los suelos en los que se realizaron las fajinas presentan unos valores más altos de materia orgánica, en comparación con zonas donde se realizaron cordones, zonas quemadas sin actuación y zonas sin quemar. En relación con la vegetación, los resultados indican que las zonas en las que se realizaron las fajinas y cordones presentan niveles equivalentes de cobertura y aparecen similares especies herbáceas y arbustivas. Las zonas quemadas sin actuación y zonas sin quemar cercanas a la zona de estudio presentan menores niveles de cobertura y aparecen especies diferentes. La diversidad vegetal y la riqueza florística son similares comparando las cuatro zonas de estudio. El presente trabajo demuestra la importancia de las medidas de restauración para recuperar la materia orgánica y los nutrientes del suelo y la vegetación después de incendio.

Palabras clave: Fajinas, acordonado, incendio forestal, calidad suelos, riqueza especies.

\section{ABSTRACT}

Postfire restoration practices encompass those, which aim to reduce negative wildfire impacts and to improve burned area rehabilitation. Soil and vegetation are highly affected by wildfires due to direct effect and 
subsequent erosion processes. This work aims to evaluate the effect on soil functionality and vegetation recovery after wildfire of two different soil restoration techniques (Contour-felled log erosion barrier and contourfelled log debris) used at slope scale and after 5 years of the wildfire event. Postfire management strategies were applied between 3 and 5 months after the fire in four areas and in triplicate (log erosion barriers, courtour felled log debris, burn and not action and unburned). Results showed similar species coverage and grass or shrubs species. Wildfire affected areas without any intervention and unaffected areas by fire presented different grass or shrubs species (but pioneer species) and lower species coverage. Species diversity and richness were similar comparing all the study plots. Our results also indicate that plots under Contour-felled log erosion barriers presented higher soil organic matter in comparison to plots under contour-felled log debris, wildfire-affected areas without any intervention or unaffected areas by fire. Postfire restoration practices generate microbiological soil properties recovery, reaching similar values to those found at control plots. This work highlights that postfire restoration practices are important for recovering soil organic matter and nutrients and vegetation after wildfires.

Keywords: Contour-felled log erosion barrier; contour-felled log debris; forest fires; soil quality; species richness.

\section{INTRODUCCIÓN}

Los trabajos de restauración post-incendio que se realizan en zonas quemadas deben estar recogidas en un plan de restauración, que tendrá unos objetivos establecidos dependiendo de la severidad del incendio, los regímenes de precipitación de la zona y de los objetivos de gestión forestal de la superficie incendiada (Alloza et al., 2014). Cada una de las actuaciones realizadas debe ser evaluada tras su ejecución en términos de eficacia (capacidad para conseguir el objetivo fijado), eficiencia (capacidad de optimizar los recursos empleados en la realización de la actuación) y efectividad (capacidad de seleccionar la actuación más correcta y realizarla de la mejor manera posible). Para ello es fundamental la investigación 
científica, que cuantifique e identifique los efectos a distintos niveles de cada una de las actuaciones.

El paso del fuego da lugar a una compleja respuesta de los suelos y la vegetación, que dependerá tanto de las características del fuego, básicamente severidad e intensidad, como a las propiedades del suelo y la vegetación dependientes del ecosistema unido al clima de la zona (Mataix-Solera y Cerdá, 2009). No obstante, el conocimiento tanto de los cambios en las propiedades de la vegetación y suelo ocasionadas por un incendio, así como el efecto de las tareas de restauración sobre el suelo y la vegetación es fundamental para los gestores, a fin de detectar aquellas actuaciones que puedan ocasionar un deterioro del ecosistema mayor al provocado por el propio incendio o compensaciones que puedan favorecer o acelerar la recuperación.

A nivel ladera, la corta de árboles quemados y la disposición de los troncos siguiendo curvas de nivel y fijados al suelo con estacas o los tocones de los propios árboles se conoce con el nombre de fajina. El acordonado corresponde con la disposición siguiendo curvas de nivel de restos de la copa y ramas de los árboles. Las fajinas o acordonados vegetales son una actuación post-incendio ampliamente extendida en el mundo forestal, como medida para el control de los procesos erosivos (escorrentía y erosión) (Robichaud et al., 2008). Además, se trata de una medida de gestión del combustible muerto quemado, que facilita el posterior manejo y uso del monte, permitiendo la descomposición e incorporación del material procesado más rápidamente al suelo con la consiguiente disminución de carga de combustible y el aumento de los posibles efectos beneficiosos sobre el suelo. Estudios previos han puesto de manifiesto resultados contradictorios dadas las diferencias existentes en el diseño de la estructura, la estación ecológica, severidad de fuego y momento de instalación. En algunos casos, el impacto de las fajinas o acordonado como elemento reductor de escorrentías y de depósitos arrastrados se limita a episodios de lluvia de poca entidad, y en todo caso siempre condicionado a su correcta instalación (e.g. Robichaud et al., 2008). Así por ejemplo se indica en Badía et al. (2015), en cuyo estudio se demuestra que la erosion se redujo de 8.1 $\mathrm{Mg} \mathrm{ha}^{-1}$ año a $0.8 \mathrm{Mg} \mathrm{ha}^{-1} \mathrm{año}^{-1}$. En otros casos, los efectos registrados son inapreciables o escasos principalmente debido a una deficiencia en su 
construcción (Robichaud et al., 2008). Finalmente, algunos estudios han puesto de manifiesto un efecto negativo, en términos de mayor porcentaje de suelo desnudo y pedregosidad, en zonas de solana sometidas a distintos tratamientos selvícolas, que podría asociarse al trasiego por la zona quemada y posterior aumento de la erosión del suelo (Raftoyanis y Spano, 2005). En el caso de la vegetación, los estudios disponibles y trabajos realizados son escasos y no concluyentes. Diferentes autores demuestran una mejora de la humedad del suelo que favorece el establecimiento de la vegetación, si bien es verdad que, a pesar del éxito inicial en la emergencia de la vegetación, la abundancia y recuperación es lenta (Rey et al., 2005). Por el contrario, Badía et al. (2017) corroboran mediante este estudio sobre el erizón (Echinospartum horridum (Vahl) Rothm.) en el pirineo Central, la rápida autosucesión vegetal después del incendio.

El objetivo del presente estudio es evaluar el efecto que las prácticas post-incendio (instalación de fajinas y acordonado) tienen sobre las propiedades fisicoquímicas y contenido en nutrientes del suelo. Como segundo objetivo se plantea evaluar el efecto de estas actuaciones post-incendio sobre la recuperación de la vegetación, en concreto, de las especies germinadoras. La hipótesis de partida es considerar que las fajinas y cordones frenan la escorrentía, acumulan sedimento y generan unas condiciones microclimáticas (sombreo) en su "área de influencia", que permiten la conservación/mejora del suelo y aceleran la sucesión vegetal. Las conclusiones de este estudio aportan información a los gestores sobre la influencia de determinadas prácticas post-incendio sobre el suelo y la vegetación.

\section{MATERIAL Y METODOS}

\subsection{Zona de estudio}

El estudio se ubica en el área afectada por el incendio forestal de la Sierra de Donceles (Hellín, Albacete), acontecido entre el 1 y el 6 de julio de 2012 y que afectó a un total de 6.500 ha de masa forestal (Figura 1; punto de ignición: 608309 E, $4254211 \mathrm{~N}$; ETRS89 UTM 30N). El incendio, iniciado al mediodía, tuvo una velocidad de propagación de 25-30 metros 
por minuto debido al viento y a la topografía, quemando en las primeras siete horas el $90 \%$ de su superficie total. La severidad del fuego en general fue alta y moderada-baja en base a las valoraciones realizadas en campo y mediante imágenes de satélite (Gómez Sánchez et al. 2017), teniendo mucho que ver en este resultado, la estructura de vegetación predominante antes del incendio. La zona pertenece a la cuenca hidrográfica del Segura estando encajada entre los ríos Mundo y Segura, al norte y al sur respectivamente, siendo el primero el afluente más caudaloso del segundo. Geológicamente la cuenca se encuentra dentro de las cordilleras Béticas, más concretamente en la zona Prebética. Los materiales que conforman esta zona son en su mayoría calizas más o menos dolomitizadas, margas y arcillas. El contenido de materia orgánica en los suelos de la zona es muy bajo en los horizontes más superficiales, situándose en torno a un $4 \%$ en las parcelas control. La altitud de la zona incendiada se encuentra entre los $314 \mathrm{~m}$ y los $808 \mathrm{~m}$ sobre el nivel del mar. La precipitación anual media de la zona de estudio es de $372 \mathrm{~mm}$, distribuida con dos máximos en octubre $(48 \mathrm{~mm}$ ) y en mayo $(47 \mathrm{~mm})$ y un mínimo en junio con $8 \mathrm{~mm}$. La temperatura media es de $16^{\circ} \mathrm{C}$, con una $\mathrm{T}^{\text {a }}$ media de las máximas de $40^{\circ}$ (máxima absoluta de $46^{\circ}$ en julio) y una $\mathrm{T}^{\mathrm{a}}$ media de las mínimas de $-2{ }^{\circ} \mathrm{C}$ (mínima absoluta de $-14^{\circ} \mathrm{C}$ en febrero). El clima se clasifica como mediterráneo subárido (Allué, 1990). La vegetación de la zona de estudio estaba dominada por pinares de pino carrasco (Pinus halepensis M.) de origen natural en zonas de vaguada, umbrías y/o zonas inaccesibles por la pendiente y pinares procedentes de repoblaciones de escaso desarrollo realizadas hace 25 años por la administración forestal. Los matorrales presentes que acompañan a los pinares están compuestos por vegetación principal o acompañante del pino carrasco, tipo espartales (Helictotricho filifolii-Stipetum tenacissimae), romerales (Anthyllido cytisoidis-Cistetum Clusii), espartales-romerales (mezcla de las anteriores) y tomillares (Anthyllido onobrychoidis-Thymetum funkii).

\subsection{Diseño del experimento}

Para la realización del estudio se seleccionó una cuenca hidrográfica de $3 \mathrm{~km}^{2}$ con distintos trabajos de restauración post incendio (Tabla 1 y 
Figura 2). Dentro de la cuenca, se identifican tres zonas donde la cubierta vegetal fue totalmente arrasada por el fuego acontecido en 2012 pero en las que se llevaron a cabo distintos tipos de trabajos post-incendio, ejecutados inmediatamente después del fuego (fajinas, acordonado, no actuación), y una cuarta zona donde la cubierta arbórea no fue afectada por el fuego. En cada zona se señalaron tres parcelas de muestreo distribuidas aleatoriamente y separadas para evitar problemas de pseudoreplicación, con un total de 12 parcelas (Tabla 1) caracterizando vegetación, cobertura, pedregosidad y profundidad de suelo. La exposición de todas las parcelas fue principalmente norte, con $550 \mathrm{~m}$ de altitud media y $25 \%$ de pendiente media, con características litológicas comparables. Antes del incendio, la vegetación presente era pinar de carrasco (con fracción de cabida cubierta $>70 \%$ ) con sotobosque de arbustivas termófilas descritas en el párrafo anterior.

\subsection{Propiedades fisicoquímicas y muestreos de vegetación.}

En junio de 2016 se recogieron tres muestras de suelo dispersas al azar dentro de cada parcela, procedentes de los primeros $10 \mathrm{~cm}$ de suelo previa retirada del material vegetal que cubría el suelo (hojarasca, restos de plantas, etc.). Cada una de las muestras se llevó inmediatamente a laboratorio donde se analizó textura, contenido de materia orgánica, $\mathrm{pH}$, conductividad eléctrica y la relación $\mathrm{C} / \mathrm{N}$. También se obtuvo el contenido en nutrientes disponibles ( $\mathrm{P}$ total, $\mathrm{Ca}, \mathrm{Na}$ y $\mathrm{K}$ ). La metodología seguida para los análisis fue la expuesta en Gómez-Sánchez et al., (2019). En las mismas parcelas, se realizaron tres transectos de vegetación (de 10 metros de longitud) por parcela en los que se muestrearon las especies vegetales existentes (sólo se consideraron en el estudio las especies germinadoras ya que se pretende ver el efecto de los trabajos sobre el reclutamiento de especies procedentes de semilla) y se calcularon la riqueza de especies, la abundancia, el índice de riqueza de Margalef, el índice de Shannon y el índice de Simpson por los procedimientos y formulaciones habituales y expuestos en la literatura científica relacionada. 


\subsection{Análisis estadístico}

Se realizó un análisis estadístico ANOVA de los datos, en concreto el denominado clasificación única del análisis de la varianza (ANOVA de una vía), considerando el tipo de tratamiento (acordonado, fajina, quemado sin actuación y no quemado) como el principal factor. Previamente, el diseño del muestreo permitió considerar todas las muestras como espacialmente independientes; comprobando la normalidad y homocedasticidad de los datos mediante test de Shapiro Wilk y test de Barlett respectivamente, realizando la transformación de las variables mediante la raíz cuadrada cuando fue necesario. Como test de comparación posthoc se empleó el Test de Tukey. Se estableció un nivel de significación en todas las pruebas del $5 \%(\mathrm{P}<0,05)$ para rechazar la hipótesis nula (las muestras pertenecen a la misma población, procediendo las diferencias entre muestras al muestreo), salvo que se indique lo contario en cada caso. Finalmente se realizó un análisis de similaridades (ANOSIM) con las especies vegetales encontradas en base al factor tratamiento.

\section{RESULTADOS Y DISCUSIÓN}

De acuerdo a los resultados, las propiedades del suelo y la vegetación han variado significativamente entre los tratamientos post-incendio a nivel ladera. Patrones significativamente diferentes en los parámetros medidos en suelo y vegetación han sido observados, lo cual induciría a pensar que los tratamientos post-incendio a nivel ladera, tienen un efecto significativo sobre el suelo y la vegetación. Los valores analizados para cada una de las condiciones experimentales pueden verse en la Tabla 2. La textura del suelo en la zona puede clasificarse como franca o franco arenosa (Clasificación USDA), sin que el paso del fuego ocasione un cambio de clase textural, aunque si induce pequeños cambios en sus fracciones. La fracción arcilla es la que se ve más afectada, disminuyendo entre un $3 \%$ y un $8 \%$ según tratamientos, correspondiendo la disminución mayor al tratamiento cordón seguido del tratamiento fajina y del no tratamiento; existiendo diferencias significativas entre grupos. Dada la homogeneidad de las condiciones previas en relación al suelo, todo indicaría que 
los cambios estarían generados por las actuaciones post-fuego. La fracción de arena no parece verse afectada, sin diferencias entre tratamientos: mientras que el limo aumenta ligeramente en las zonas quemadas, con un aumento significativo en el caso de las parcelas donde se ha efectuado el tratamiento cordón. Tras un incendio forestal, las diferentes fracciones de textura de un suelo se verán afectadas siempre que se alcancen temperaturas superiores a $400^{\circ} \mathrm{C}$, en cuyo caso la repuesta de cada fracción será distinta (Muñoz Rojas et al., 2015). De aquí las diferencias en los resultados encontrados. Además, la profundidad del suelo y la inercia térmica del mismo, genera cambios en los efectos que el suelo genera sobre las propiedades edáficas (Badía y Martí, 2003).

Las parcelas quemadas con tratamiento presentan mayor contenido en materia orgánica que las no tratadas, con un aumento de más del 3\% de media para el tratamiento fajina y del $2 \%$ para el cordón. Existen dos grupos que presentan diferencias significativas en sus medias, uno es el compuesto por las parcelas no quemadas (control) y las parcelas quemadas sin tratamiento, frente a las parcelas donde se ha realizado tratamiento de fajina y cordón. Este hecho puede estar generado por dos factores: el efecto barrera/deposición de suelo realizado por los cordones y fajinas, y la lenta incorporación de restos procedentes del material vegetal utilizado en su construcción, que favorecerían el aumento de la materia orgánica del suelo (Gómez-Sánchez et al., 2019). La relación C/N se sitúa entre 10 de las parcelas control y 16 para el tratamiento cordón, con diferencias significativas entre ambos grupos. Los resultados indican que, en ausencia de tratamiento post-incendio, cinco años tras el fuego, la MO y la relación $\mathrm{C} / \mathrm{N}$ tienden al equilibrio existente antes del fuego, desapareciendo sus efectos iniciales. Por el contrario, el manejo post-incendio altera dicho equilibrio y produce un incremento en la cantidad de materia orgánica y mejora la relación $\mathrm{C} / \mathrm{N}$ de los suelos, lo que supone una mejora de la calidad edáfica. Las fajinas son el tratamiento que induce mayor aumento de materia orgánica, lo que es de esperar dada la deposición de restos aguas arriba de las mismas.

Los valores de $\mathrm{pH}$ son altos en todos los grupos (entre 8,2 -8,5), si bien es verdad que el $\mathrm{pH}$ para el tratamiento cordón muestra diferencias con el resto de tratamientos salvo con las fajinas, de manera que los tra- 
tamientos en ladera disminuirían en una pequeña proporción los valores de $\mathrm{pH}$ del suelo. Los valores de CE son bajos para todos los tratamientos $(<0,2 \mathrm{dS} / \mathrm{cm})$, si bien se observa que, en las zonas afectadas por el fuego, la conductividad eléctrica es mayor, oscilando su media entre $160-175 \mu \mathrm{S}$ $\mathrm{cm}^{-1}$ en zonas quemadas, frente a los 124 en las parcelas control. Existen diferencias significativas en la conductividad medida de las parcelas control y la medida en parcelas quemadas con tratamiento fajina, donde la CE es más alta. Los suelos con baja conductividad eléctrica indicarían un suelo pobre en nutrientes (Smith y Doran 1996), aunque bien es verdad que los suelos forestales presentan, de forma general, alta estabilidad estructural y bajos valores de conductividad eléctrica.

La concentración de nutrientes es baja en las zonas de estudio. De los tres cationes básicos analizados, el más abundante en la zona es el Ca, alcanzado valores más elevados en las zonas afectadas por el fuego, aunque el test no detecta diferencias significativas entre grupos al igual que ocurre con el Na. La concentración de K se muestra significativamente mayor en parcelas tratadas, duplicándose en el caso de las fajinas. El contenido de fosforo en muy bajo, si bien mayor en las zonas afectadas por el fuego, y presentando diferencias significativas en el caso de las fajinas. Tras el incendio los nutrientes se depositan sobre la superficie del suelo en forma de cenizas o materia orgánica quemada, lo que supone una entrada de nutrientes, y por tanto un incremento en la fertilidad del suelo (Machado, 2015). Según los datos obtenidos, los valores de Na y Ca retornan a sus valores pre-incendio transcurridos cinco años tras el incendio, destacando el comportamiento del $\mathrm{K}$ que mantiene un incremento en la zona incendiada y en las parcelas con tratamiento de fajinas o cordones.

En el caso de la vegetación (Tabla 3, 4 y 5), los resultados indican niveles similares en los índices de riqueza de especies y de índices de biodiversidad calculados, si bien es verdad, que el número de individuos se ve reducido en las parcelas afectadas por incendio. De este modo, se puede afirmar que los trabajos de restauración favorecerían la recuperación de los niveles de riqueza y biodiversidad 5 años después del incendio ya que se alcanzan los valores de la zona no incendiada. Es interesante destacar que las obras de actuación post-incendio presentan buenos regenerados de pino carrasco, lo que indicaría que la respuesta de esta especie arbó- 
rea es óptima después de incendio y no necesita apoyarse en medidas de restauración para lograr su reclutamiento inicial después de incendio. En general, las especies arbustivas y herbáceas aparecidas son similares a lo largo de las parcelas de estudio (Tabla 4). Por último, en la Tabla 5 se muestran las principales especies que contribuyen a la disimilitud de las zonas de actuación en base a las especies encontradas en cada uno de los inventarios de vegetación. Tal y como demostró el análisis ANOSIM, la disimilaridad más baja se detecta entre las parcelas de fajinas y acordonado (Tabla 5), y las especies que contribuyen a ella son principalmente Cistus albidus L., Pinus halepensis M. y Rhamnus lycioides L.. En el lado opuesto, las parcelas que presentan una mayor disimilaridad son las parcelas quemadas sin actuación y las parcelas no quemadas, contribuyendo a esta diferenciación principalmente Cistus albidus L., Halimium halimifolium L. y Pinus halepensis M.. Será interesante observar la evolución del ecosistema en el medio plazo, para ver si las diferencias encontradas entre las zonas de actuación, dan lugar a comunidades vegetales más y mejor diferenciadas.

\section{CONCLUSIONES}

Los trabajos de restauración post-incendio a nivel ladera realizados (cordones y fajinas) entre 3 y 5 meses después el incendio mejorar los contenidos de materia orgánica y nutrientes del suelo cinco años después del incendio. El efecto barrera introducido por las obras de restauración unido a la descomposición de las estructuras que poco a poco favorecería la incorporación de restos vegetales y madera quemada al suelo, lo que se refleja en los cambios en las propiedades edáficas medidas. En relación a la vegetación y siempre referido a especies germinadoras, se han encontrado valores similares de riqueza e índices de biodiversidad, lo que indicaría que las actuaciones de restauración favorecen la recuperación de la vegetación, llegando a los niveles e biodiversidad existentes en zonas no incendiadas. En general, las especies arbustivas y herbáceas aparecidas son similares a lo largo de las parcelas de estudio y tratamientos aplicados. El presente trabajo pone de manifiesto la importancia de la aplicación de las medidas de emergencia después de incendio, si bien es verdad, que se debe profundizar y seguir con estudios a más largo plazo. 


\section{AGRADECIMIENTOS}

Los autores agradecen a los servicios forestales de la Junta de Comunidades de Castilla La Mancha, Servicios Periféricos de la Consejería de Agricultura en Albacete, por la ayuda y asistencia en los trabajos de campo. El trabajo presentado en esta comunicación ha sido financiado por los proyectos "Mejora de la resiliencia y disminución de la vulnerabilidad de los ecosistemas forestales afectados por incendios forestales en ambientes mediterráneos (REVULFO)" financiado por la Diputación de Albacete y la Universidad de Castilla La Mancha y por el proyecto "Reducción de la Severidad del Fuego Mediante Nuevas Herramientas y Tecnologías para la Gestión Integrada de la Protección contra los Incendios Forestales “GEPRIF” (RTA2014-00011-C06-05)" financiado por el Ministerio de Economía y Competitividad, INIA.

\section{BIBLIOGRAFÍA}

Alloza J, García S, Gimeno T, Baeza M, Vallejo V. (2014). Guía técnica para la gestión de montes quemados. Madrid: Ministerio de Agricultura, Alimentación y Medio Ambiente.

Allué J. (1990). Atlas fitoclimático de España. Taxonomías. Ministerio de Agricultura, Pesca y Alimentación. INIA Madrid.

Badía, D., Martí, C. (2003). Plant Ash and Heat Intensity Effects on Chemical and Physical Properties of Two Contrasting Soils, Arid Land Research and Management, 17:1, 23-41.

Badía D., Sánchez C., Aznar J.M., Martí C. (2015). Post-fire hillslope $\log$ debris dams for runoff and erosion mitigation in the semiarid Ebro Basin. Geoderma, 237: 298-307.

Badía, D., Armas Herrera, C., Mora Hernández, J.L., Gómez, D., Montserrat, G., Palacios, S. (2017). ¿Podemos controlar la expansión del erizón mediante quemas? Lucas Mallada: revista de ciencias, 19, 69-94.

Gómez-Sánchez, E., De Las Heras, J., Lucas-Borja, M., Moya, D. (2017). Ajuste de metodologías para evaluar severidad de quemado en zonas semiáridas (SE peninsular): incendio Donceles 2012. Rev. Teledetección 103-113. 
Gómez-Sánchez E, Lucas-Borja Me, Plaza-Álvarez Pa, GonzálezRomero J, Sagra J, Moya D, De Las Heras J. (2019). Effects of postfire hillslope stabilisation techniques on chemical, physico-chemical and microbiological soil properties in Mediterranean forest ecosystems. Journal of Environmental Management. 246, 229-238.

Machado, A.I., Serpa, D., Ferreira, R.V., Rodríguez-Blanco, Pinto, R., Nunes, M.., Cerqueira, M.A., Keizer, J. (2015). Cation export by overland flow in a recently burnt forest area in north-central Portugal. Sci. Total Environ. 524-525, 201-212.

Mataix-Solera J, Cerdà A. (2009). Incendios forestales en España. Ecosistemas terrestres y suelos. Efectos de los incendios forestales sobre los suelos en España. El estado de la cuestión visto por los científicos españoles. FUEGORED, Cátedra Divulgación de la Ciencia, Universitat de Valencia, Spain, 27-53.

Muñoz-Rojas M, Erickson TE, Martini D, Dixon KW, Merritt DJ (2016). Soil physicochemical and microbiological indicators of short, medium and long term post-fire recovery in semi-arid ecosystems. Ecological indicators; 63: 14-22.

Raftoyannis Y., Spanos I. (2005). Evaluation of log and branch barriers as post-fire rehabilitation treatments in a Mediterranean pine forest in Greece. International Journal of Wildland Fire; 14: 183-188.

Rey F.A, Isselin-Nondedeu, F., Bedecarrats, A. (2016). Vegetation dynamics on sediment deposits upstream of bioengineering works in mountainous marly gullies in a Mediterranean climate (Southern Alps, France). Plant and Soil 278: 149-158.

Robichaud PR, Lewis S, Ashmun L. (2008). New procedure for sampling infiltration to assess post-fire soil water repellency: US Department of Agriculture, Forest Service, Rocky Mountain Research Station.

Smith J, Doran J. (1996). Measurement and use of $\mathrm{pH}$ and electrical conductivity for soil quality analysis. Methods for assessing soil quality. 49. Soil Science Society of America Madison, WI. 
Tabla 1. Tipo, cobertura vegetal, pedregosidad y cubierta vegetal de cada parcela.

\begin{tabular}{|c|c|c|c|c|}
\hline Zona & Tipo & Parcela & $\begin{array}{c}\text { Cobertura } \\
\qquad \%)\end{array}$ & $\begin{array}{c}\text { Pedregosidad } \\
\text { superficial } \\
(\%)\end{array}$ \\
\hline \multirow{3}{*}{ A } & \multirow{3}{*}{$\begin{array}{c}\text { No } \\
\text { Quemada }\end{array}$} & 10 & 95 & $50-60$ \\
\hline & & 11 & 100 & 70 \\
\hline & & 12 & 80 & 90 \\
\hline \multirow{3}{*}{ B } & \multirow{3}{*}{$\begin{array}{l}\text { Quemada } \\
\text { No } \\
\text { actuación }\end{array}$} & 7 & $70-75$ & 70 \\
\hline & & 8 & 80 & 85 \\
\hline & & 9 & 80 & 70 \\
\hline \multirow{3}{*}{$\mathrm{C}$} & \multirow{3}{*}{$\begin{array}{c}\text { Quemada } \\
\text { Cordón }\end{array}$} & 4 & 80 & 80 \\
\hline & & 5 & 80 & 70 \\
\hline & & 6 & $80-85$ & $50-60$ \\
\hline \multirow{3}{*}{$\mathrm{D}$} & \multirow{3}{*}{$\begin{array}{l}\text { Quemada } \\
\text { Fajina }\end{array}$} & 1 & $89-90$ & 80 \\
\hline & & 2 & $50-60$ & 60 \\
\hline & & 3 & 70 & $80-90$ \\
\hline
\end{tabular}

Tabla 2. Propiedades fisicoquímicas y contenido de nutrientes de suelos $(0-10 \mathrm{~cm})$ bajo diferentes tratamientos $(n=3)$. Condiciones experimentales con diferente letra indican diferencias significativas según el test de Tukey $(\mathrm{P}<0.05)$.

\begin{tabular}{ccccc}
\hline & \multicolumn{4}{c}{ Tratamiento } \\
\cline { 2 - 4 } & No quemado & Quemado sin act. & Acordonado & Fajina \\
Arcilla $(\%)$ & $9,7 \pm 0,1 \mathrm{~d}$ & $6,3 \pm 0,4 \mathrm{c}$ & $1,1 \pm 0,7 \mathrm{a}$ & $3,3 \pm 1,8 \mathrm{~b}$ \\
Limo $(\%)$ & $38,3 \pm 0,1 \mathrm{a}$ & $41,0 \pm 8,9 \mathrm{a}$ & $49,5 \pm 2,7 \mathrm{~b}$ & $41,6 \pm 5,1 \mathrm{a}$ \\
Arena $(\%)$ & $51,9 \pm 0,1 \mathrm{a}$ & $52,5 \pm 8,7 \mathrm{a}$ & $49,3 \pm 1,9 \mathrm{a}$ & $55,0 \pm 6,8 \mathrm{a}$ \\
$\mathrm{MO}(\%)$ & $4,0 \pm 0,9 \mathrm{a}$ & $4,0 \pm 0,8 \mathrm{a}$ & $6,0 \pm 0,9 \mathrm{~b}$ & $7,3 \pm 1,0 \mathrm{~b}$ \\
$\mathrm{pH} 1: 2.5$ & $8,5 \pm 0,2 \mathrm{a}$ & $8,4 \pm 0,2 \mathrm{a}$ & $8,3 \pm 0,2 \mathrm{~b}$ & $8,3 \pm 0,2 \mathrm{ab}$ \\
$\mathrm{CE} 1: 5\left(\mu \mathrm{Scm} \mathrm{cm}^{-1}\right)$ & $123,7 \pm 11,9 \mathrm{a}$ & $165,7 \pm 11,52 \mathrm{ab}$ & $160,2 \pm 10,22 \mathrm{ab}$ & $175,6 \pm 11,5 \mathrm{~b}$ \\
$\mathrm{C} / \mathrm{N}$ & $11,8 \pm 1,0 \mathrm{a}$ & $13,7 \pm 1,4 \mathrm{~b}$ & $15,6 \pm 0,9 \mathrm{~b}$ & $14,3 \pm 1,1 \mathrm{ab}$ \\
$\mathrm{P}(\%)$ & $2,3 \times 10^{-3} \pm 1,3 \times 10^{-5} \mathrm{~b}$ & $3,2 \times 10^{-3} \pm 1,1 \mathrm{x} 10^{-5} \mathrm{a}$ & $4,2 \times 10^{-3} \pm 1,4 \times 10^{-5} \mathrm{ab}$ & $7,5 \times 10^{-3} \pm 1,3 \times 10^{-5} \mathrm{~b}$ \\
$\mathrm{Ca}(\%)$ & $5,3 \pm 0,2 \mathrm{a}$ & $5,5 \pm 0,6 \mathrm{a}$ & $6,2 \pm 0,2 \mathrm{a}$ & $6,0 \pm 0,9 \mathrm{a}$ \\
$\mathrm{Na}(\%)$ & $0,5 \pm 0,1 \mathrm{~b}$ & $0,5 \pm 0,08 \mathrm{~b}$ & $0,4 \pm 0,1 \mathrm{~b}$ & $0,6 \pm 0,1 \mathrm{a}$ \\
$\mathrm{K}(\%)$ & $0,1 \pm 0,05 \mathrm{a}$ & $0,2 \pm 0,1 \mathrm{~b}$ & $0,3 \pm 0,1 \mathrm{~b}$ & $0,4 \pm 0,1 \mathrm{~b}$ \\
\hline
\end{tabular}


Tabla 3. Especies vegetales existentes (sólo se consideraron en el estudio las especies germinadoras), la riqueza de especies, la abundancia, el índice de riqueza de Margalef, el índice de Shannon y el índice de Simpson en cada una de los tratamientos estudiados. Condiciones experimentales con diferente letra indican diferencias significativas según el test de Tukey $(\mathrm{P}<0.05)$.

\begin{tabular}{lcccc}
\hline & No quemado & Quemado sin act. & Acordonado & Fajina \\
Riqueza de especies & $7 \pm 1 \mathrm{a}$ & $6 \pm 1 \mathrm{a}$ & $5 \pm 2 \mathrm{a}$ & $6 \pm 1 \mathrm{a}$ \\
Individuos & $608 \pm 204 \mathrm{~b}$ & $371 \pm 114 \mathrm{a}$ & $455 \pm 200 \mathrm{ab}$ & $295 \pm 131 \mathrm{a}$ \\
Riqueza Margalef & $0.7 \pm 0.1 \mathrm{a}$ & $0.8 \pm 0.04 \mathrm{~b}$ & $0.8 \pm 0.1 \mathrm{~b}$ & $0.8 \pm 0.08 \mathrm{~b}$ \\
Shannon & $1.4 \pm 0.2 \mathrm{a}$ & $1.6 \pm 0.1 \mathrm{a}$ & $1.4 \pm 0.3 \mathrm{a}$ & $1.4 \pm 0.2 \mathrm{a}$ \\
Simpson & $0.6 \pm 0.1 \mathrm{a}$ & $0.76 \pm 0.04 \mathrm{a}$ & $0.6 \pm 0.1 \mathrm{a}$ & $0.71 \pm 0.07 \mathrm{a}$ \\
\hline
\end{tabular}

Tabla 4. Porcentaje de abundancia de las principales especies germinadoras encontradas en cada condición experimental.

\section{\% abundancia}

\section{G1: No quemado}

Cistus albidus L. 36,5

Pinus halepensis M. 22,1

Halimium halimifolium L. Willk. $\quad 14,6$

Serratula leucantha (Cav.) DC $\quad 11,2$

Rosmarinus officinalis L. $\quad 5,5$

\section{G2: Quemado sin actuación}

Fumana ericoides (Cav.) Gand. 27,2

Pinus halepensis M. 21,4

Rosmarinus officinalis M. 20,7

Cistus clusii Dunal. 15,1

Helianthemum cinereum (Cav.) $\quad 5,6$

G3: Quemado y Acordonado

Cistus albidus L. 38,2

Pinus halepensis M. $\quad 16,3$

Cistus clusii Dunal 15,3

Rosmarinus officinalis L. $\quad 9,6$

Helianthemum cinereum (Cav.) $\quad 8,3$

Rhamnus lycioides L. $\quad 6,0$ 


\section{G4: Quemado y con Fajinas}

\begin{tabular}{lc} 
Rosmarinus officinalis L. & 31,1 \\
Cistus albidus L. & 25,0 \\
Pinus halepensis M. & 13,7 \\
Cistus clusii Dunal & 13,4 \\
Fumana ericoides (Cav.) Gand & 5,2 \\
Centaurea antennata Dufour & 4,8 \\
\hline
\end{tabular}

Tabla 5. Porcentaje de abundancia de las principales especies encontradas en cada condición experimental.

\begin{tabular}{|c|c|c|c|c|}
\hline G1 and G2: Media disimilaridad =70.99 & Media G1 & Media G2 & Contribución\% & Cum.\% \\
\hline Cistus albidus L. & 208.33 & 0 & 28.21 & 28.21 \\
\hline Halimium halimifolium L. Willk. & 115 & 25.78 & 15.83 & 44.04 \\
\hline Pinus halepensis M. & 100 & 68.44 & 10.31 & 54.35 \\
\hline G1 and G3: Media disimilaridad =65.59 & Media Gl & Media G3 & Contribución\% & Cum.\% \\
\hline Cistus albidus L. & 208.33 & 170.56 & 26.71 & 26.71 \\
\hline Halimium halimifolium L. Willk. & 115 & 30.78 & 16.42 & 43.14 \\
\hline Pinus halepensis M. & 100 & 59.11 & 10.94 & 54.08 \\
\hline G2 and G3: Media disimilaridad $=68.55$ & Media G2 & Media G3 & Contribución\% & Cum.\% \\
\hline Cistus albidus L. & 0 & 170.56 & 27.57 & 27.57 \\
\hline Fumana ericoides (Cav.) Gand & 76.44 & 4.44 & 13.14 & 40.7 \\
\hline Pinus halepensis M. & 68.44 & 59.11 & 10 & 50.7 \\
\hline G1 and G4: Media disimilaridad =71.46 & Media G1 & Media G4 & Contribución\% & Cum.\% \\
\hline Cistus albidus L. & 208.33 & 89.67 & 27 & 27 \\
\hline Halimium halimifolium $\mathrm{L}$. & 115 & 6.11 & 17.56 & 44.56 \\
\hline Pinus halepensis M & 100 & 33.56 & 12.34 & 56.9 \\
\hline G2 and G4: Media disimilaridad =66.48 & Media G2 & Media G4 & Contribución\% & Cum.\% \\
\hline Cistus albidus M. & 0 & 89.67 & 18.08 & 18.08 \\
\hline Fumana ericoides (Cav.) Gand & 76.44 & 14.11 & 14.63 & 32.71 \\
\hline Pinus halepensis M. & 68.44 & 33.56 & 12.7 & 45.41 \\
\hline G3 and G4: Media disimilaridad =63.93 & Media G3 & Media G4 & Contribución\% & Cum.\% \\
\hline Cistus albidus L. & 170.56 & 89.67 & 30.89 & 30.89 \\
\hline Pinus halepensis M. & 59.11 & 33.56 & 9.94 & 40.83 \\
\hline Rhamnus lycioides L. & 41.11 & 30 & 9.83 & 50.66 \\
\hline
\end{tabular}


Figura 1. Zona de Estudio.
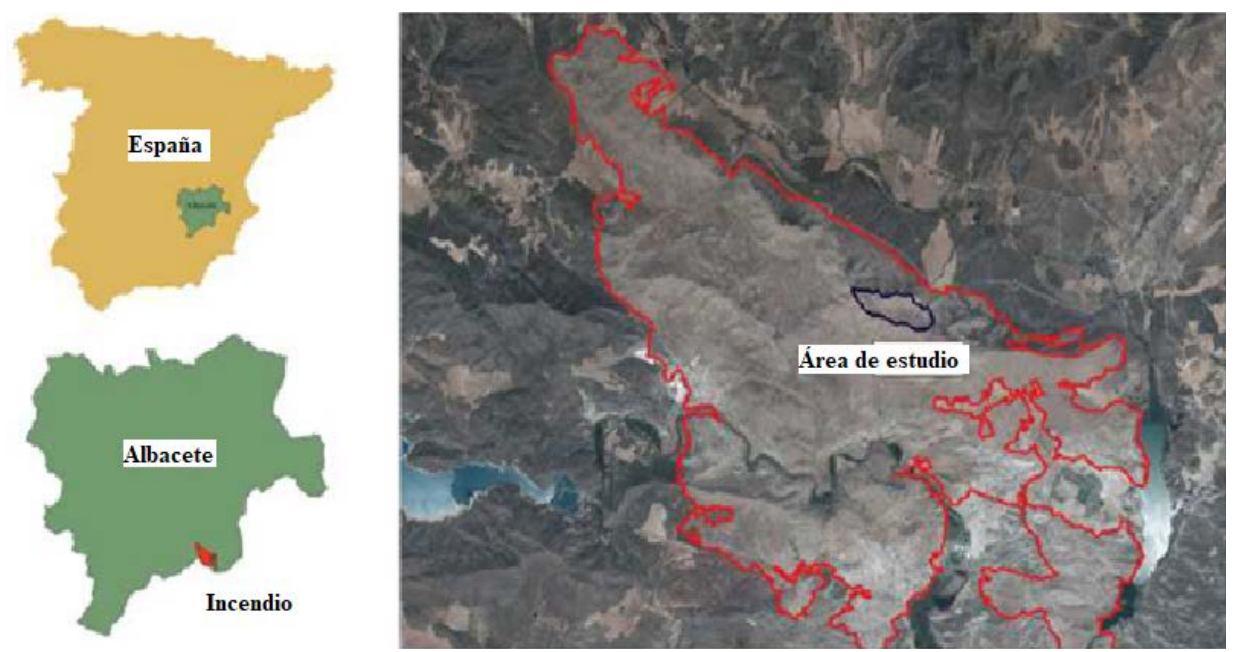

Figura 2. Fotografías de las diferentes zonas de estudio un año después del incendio.

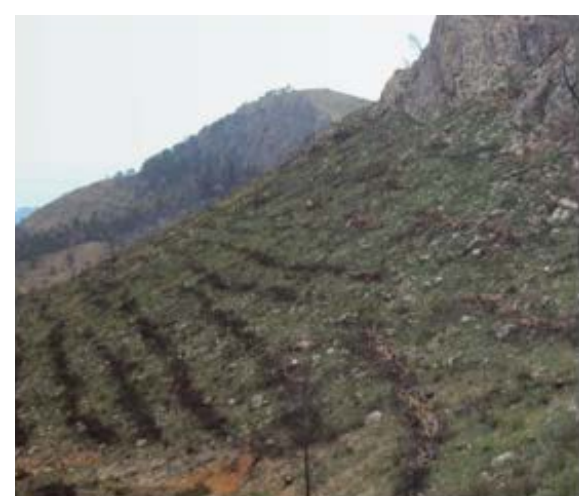

Acordonado

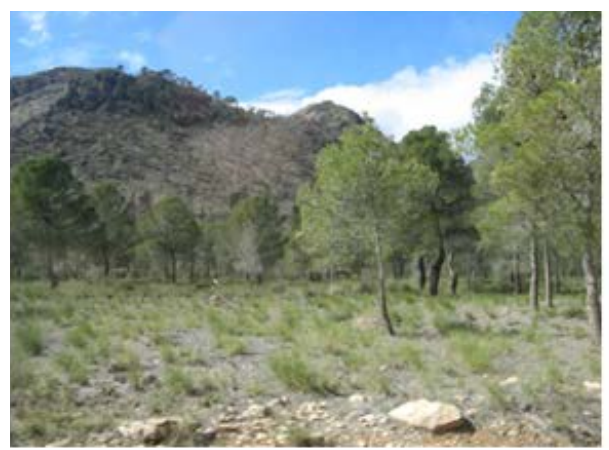

Control

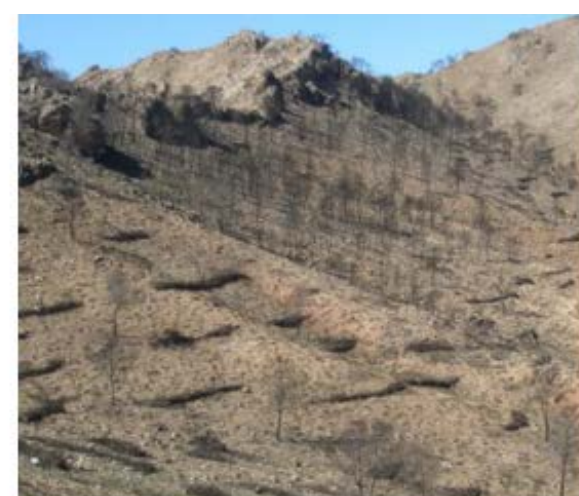

Fajinas

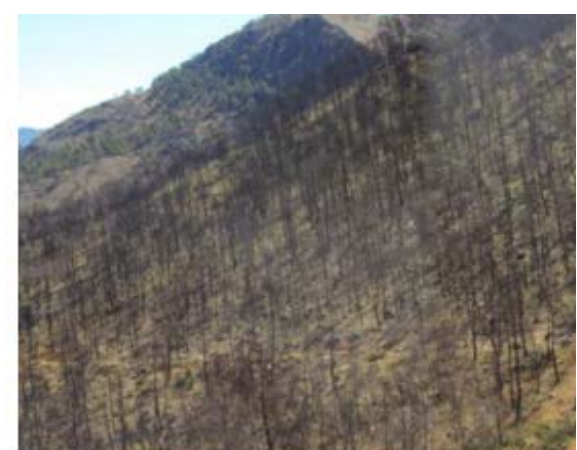

Quema sin actuación 